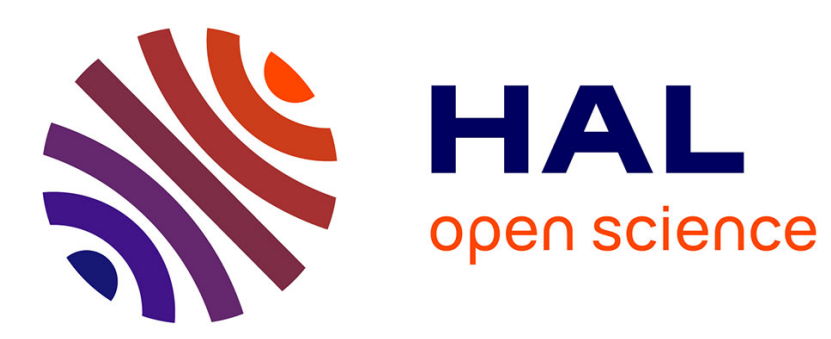

\title{
Simulation of Dicke-narrowed molecular spectra recorded by off-axis high-finesse optical cavities
}

\author{
Pasquale Maddaloni, Pietro Malara, Paolo de Natale
}

\section{To cite this version:}

Pasquale Maddaloni, Pietro Malara, Paolo de Natale. Simulation of Dicke-narrowed molecular spectra recorded by off-axis high-finesse optical cavities. Molecular Physics, 2010, 108 (06), pp.749-755. 10.1080/00268971003601571 . hal-00588665

\section{HAL Id: hal-00588665 https://hal.science/hal-00588665}

Submitted on 26 Apr 2011

HAL is a multi-disciplinary open access archive for the deposit and dissemination of scientific research documents, whether they are published or not. The documents may come from teaching and research institutions in France or abroad, or from public or private research centers.
L'archive ouverte pluridisciplinaire HAL, est destinée au dépôt et à la diffusion de documents scientifiques de niveau recherche, publiés ou non, émanant des établissements d'enseignement et de recherche français ou étrangers, des laboratoires publics ou privés. 


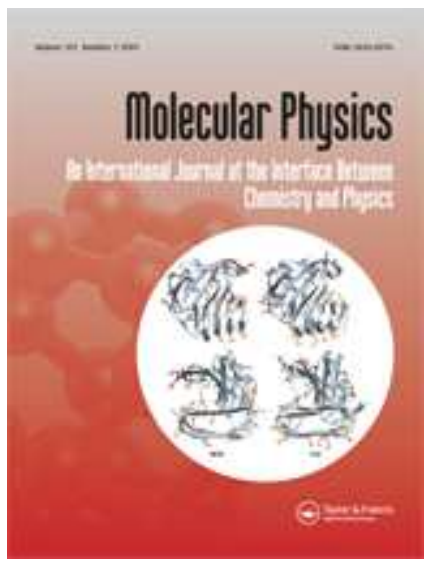

\section{Simulation of Dicke-narrowed molecular spectra recorded by off-axis high-finesse optical cavities}

\begin{tabular}{|r|l|}
\hline Journal: & Molecular Physics \\
\hline Manuscript ID: & TMPH-2009-0336.R1 \\
\hline Manuscript Type: & $\begin{array}{l}\text { Special Issue Paper -HRMS Stabia 09/ High Resolution Molecular } \\
\text { Spectroscopy }\end{array}$ \\
\hline Date Submitted by the \\
Author: & $14-$ Dec-2009 \\
\hline Complete List of Authors: & $\begin{array}{l}\text { maddaloni, pasquale; Istituto Nazionale di Ottica Applicata, } \\
\text { Consiglio Nazionale delle Ricerche } \\
\text { de natale, paolo; Istituto Nazionale di Ottica Applicata }\end{array}$ \\
\hline Keywords: & molecular profiles, high-finesse optical cavities, Dicke narrowing \\
\hline
\end{tabular}

\section{S ScholaroNE \\ Manuscript Central}




\begin{abstract}
We present simulations of molecular spectral profiles recorded in Dicke narrowing regime by off-axis high-finesse optical cavities. The influence of experimental parameters on the transmitted lineshapes is investigated on the basis of a model which takes into account the effect of the cavity transfer function. In particular, the collisional narrowing pressure range is found to shrink until disappearance as the molecular absorption increases. We also show that fitting the measured lineshapes with a pure Galatry function, i.e. neglecting instrumental effects, may lead to discrepancies of several tens percent in the extracted line parameters.
\end{abstract}

Introduction - Accurate modeling of line shapes plays a crucial role for a correct analysis of molecular spectra with particular relevance in the study of fundamental chemical processes [1], planetary atmospheres [2-4] and stellar dynamics [5, 6]. Most experimental line shapes are properly described by the Voigt profile, which is a convolution of Lorentzian and Gaussian distributions. However, this simplified model fails, for instance, in presence of collisional, or Dicke, narrowing [7]. Such effect is observed when the excited-state lifetime is long compared to the mean time between collisions. During this lapse the mean velocity of the molecule approaches zero, as the collisional averaging causes the latter to take on all possible velocities. Under collisional narrowing the line profile becomes Lorentzian-like putting on a width narrower than the Doppler one and inversely proportional to gas pressure. The specific functional form obtained for the lineshape depends on the assumptions made regarding the nature of the velocity-changing collisions and their correlation or lack thereof with collisions which perturb the internal state of the molecule [8-11]. In particular, Nelkin-Ghatak and Galatry profiles have been extensively used to determine the Dickenarrowing effect and extract fundamental line parameters from molecular spectra observed in different experimental conditions [12-18]. Meanwhile, some refined theoretical models have been proposed to describe more and more accurately collision-narrowed profiles [19, 20]. However, only few works have dealt with the effects induced on the recorded spectral profiles by the transfer function associated with the specific measurement technique. Actually, such effects are negligible when spectra are measured with small relative absorptions, but may, vice versa, become significant 
when high-sensitivity spectroscopic techniques are implemented. In this frame, for example, the capability of retrieving precise spectral information from Dicke-narrowed lineshapes recorded by two-tone frequency modulation spectroscopy has been discussed [21].

In the case of extremely weak molecular transitions, enhancing the effective interaction pathlength by means of high-finesse optical cavities may be necessary to achieve a signal-to-noise ratio that is sufficient to allow accurate lineshape fitting. Among the most valuable schemes, off-axis integrated-cavity-output spectroscopy (ICOS) offers several advantages. It combines the detection principle of cavity-enhanced absorption spectroscopy (CEAS) with a much simpler setup, using a multipasslike alignment for the high-finesse cavity [22-26]. Recently, Gupta et al. have exactly reported absorption measurements of molecular hydrogen lines in Dicke-narrowing regime performed in the near infrared $\left(8075 \mathrm{~cm}^{-1}\right)$ by off-axis ICOS [27]. Then, by fitting directly a Galatry function to the measured profiles, the effective frequency of velocity-changing collisions could be extracted.

In this paper, we theoretically investigate the influence of the off-axis ICOS transfer function on molecular spectra recorded in Dicke-narrowing regime. In particular, we find that, under typical working conditions, the pressure range exhibiting the linewidth narrowing reduces as the cavity gain increases, and eventually even disappears for sufficiently high absorptions. Also, we show that, fitting the lineshape without accounting for the cavity transfer function may lead to significant discrepancies in the extracted line parameters.

The model - The off-axis geometry, while providing an interaction path-length enhancement of $F / \pi$, (with $F$ as the cavity finesse), results in the excitation of an extremely dense mode spectrum so that the interaction between the laser and the cavity can be considered as always resonant. On the basis of this consideration, the cavity output intensity can be written as [22, 26]

$$
I_{t}(v, p)=\frac{1}{2} \cdot \frac{I_{0} \cdot C \cdot T_{m}^{2}}{1-R_{m}}
$$

where $I_{0}$ is the incident intensity, $C$ a factor between 0 and 1 describing the amount of incident radiation coupled to the resonator, and $R_{m}\left(T_{m}\right)$ the mirror reflectivity (transmittivity). Then, in presence of an absorbing species, Eq. 1 becomes

$$
I_{t}(v, p)=\frac{1}{2} \cdot \frac{I_{0} \cdot C \cdot T_{m}^{2}}{1-R_{m} \cdot e^{-\alpha(v, p) \cdot p \cdot L}}
$$

where $L$ is the cavity length, $p$ the gas pressure, and $\alpha(v, p)$ the absorption coefficient of the selected transition. Concerning the lineshape modeling, we choose a Galatry function to describe the molecular profile in the collision-narrowed regime. Such function is derived under the two 
following assumptions. First, the effects of individual collisions are negligible, so that the velocity after a single collision is strongly correlated with the velocity prior to it, and only the cumulative action of many collisions results in an isotropic velocity distribution (soft-collision model). Second, velocity-perturbing collisions are statistically independent of state-perturbing collisions. It is worth noting that the soft-collision model is more appropriate for collisions with relatively light perturbers, albeit it is not restricted to this case. On the other hand, the hard collision model, from which the Nelkin-Ghatak profile is derived, explicitly assumes that every collision completely destroys the velocity correlation. For this reason, the soft collision model can be extended to more general situations with little numerical error. Furthermore, although the lineshape functions derived from the hard and soft collision models are different, the resulting profiles are numerically quite similar, particularly when collisional broadening is also significant. Following the standardized form suggested by Herbert [10], the Galatry profile is conveniently expressed as

$$
G\left(x^{\prime}, y, z\right)=\frac{1}{\pi} \operatorname{Re}\left[\frac{1}{\frac{1}{2 z}+y-i x^{\prime}} M\left(1 ; 1+\frac{1}{2 z^{2}}+\frac{y-i x^{\prime}}{z} ; \frac{1}{2 z^{2}}\right)\right]
$$

where $M$ is the confluent hypergeometric function (note that $\int_{-\infty}^{+\infty} G\left(x^{\prime}, y, z\right) d x=1$ ). The dimensionless variables in Eq. 3 are defined as follows

$$
\begin{aligned}
& x^{\prime}=\frac{v-v_{0}}{\Delta v_{D}}-\frac{\delta}{\Delta v_{D}} p \\
& y=y_{0} \cdot p, \quad\left(y_{0}=\frac{\gamma}{\Delta v_{D}}\right) \\
& z=\frac{k_{B} \cdot T}{2 \cdot \pi \cdot c \cdot m \cdot \Delta v_{D} \cdot D}
\end{aligned}
$$

where $v_{0}$ is the line center frequency, $\Delta v_{D}$ the 1/e Doppler halfwidth, $\delta$ the line shift per unit pressure, $\gamma$ the collision width per unit pressure, $k_{B}$ the Boltzmann constant, $T$ the gas temperature, $c$ the speed of light, $m$ the molecule mass, and $D$ the so-called optical diffusion coefficient. The latter varies inversely with pressure so that Eq. 6 can be re-written as

$$
z=z_{0} \cdot p, \quad\left(z_{0}=\frac{k_{B} \cdot T}{2 \cdot \pi \cdot c \cdot m \cdot \Delta v_{D} \cdot D_{0}}\right)
$$

where $D_{0}$ is a function of temperature and some constants related to the gas species [28, 29]. Now, if we denote with $S$ the line strength and $N_{L}$ the Loschmidt number, the absorption coefficient of the selected transition takes the form 


$$
\alpha(v, p)=\frac{S \cdot N_{L}}{\Delta v_{D}} G(v, p)
$$

Finally, molecular profiles measured by off-axis ICOS are recovered by inserting Eq. 8 into Eq. 2 , so that the relative absorption signal on the cavity output is

$$
V(v, p) \equiv \frac{I_{t}(v, p=0)-I_{t}(v, p)}{I_{t}(v, p=0)}=\frac{1-e^{-\alpha(v, p) \cdot p \cdot L}}{1 / R_{m}-e^{-\alpha(v, p) p \cdot L}}
$$

From the above formula one can better appreciate the cavity enhancement effect; indeed, as the mirror reflectivity approaches 1 , the relative absorption tends to $100 \%$.

Results and discussion - In order to simulate the profile of Dicke-narrowed spectra as recorded by means of off-axis ICOS, we perform a umerical evaluation of Eq. 9. For this purpose we resort to Mathematica Software, where the hypergeometric confluent is compiled as a special function. In the following, we consider the case where the cavity is filled only with the absorbing gas (no buffer gas is present). Of course our treatment can be straightforwardly extended to more general configurations. Since Dicke narrowing can only be observed if the absorbing species has a Doppler width that is substantially greater than its pressure-broadened line width, we assume $\Delta v_{D}=0.01 \mathrm{~cm}^{-}$ ${ }^{1}(\approx 300 \mathrm{MHz})$ and $\gamma=0.001 \mathrm{~cm}^{-1} \cdot \mathrm{atm}^{-1}$, so that $y_{0}=0.1 \mathrm{~atm}^{-1}$. The order of magnitude of such values is typical, for example, of light molecules like $\mathrm{H}_{2}$ or $\mathrm{D}_{2}$ [30, 13]. Indeed, a self-broadening coefficient as low as $5 \cdot 10^{-4} \mathrm{~cm}^{-1}$ at STP was found in the first studies of Dicke narrowing carried out on the hydrogen quadrupole lines belonging to vibrational-rotational bands observed in absorption from Jupiter [2]. Concerning the line shift per unit pressure, we set $\delta=0.001 \mathrm{~cm}^{-1} \mathrm{~atm}^{-1}$ which is close to the value measured for $\mathrm{H}_{2}$ by Gupta et al. [27]. The order of magnitude of $z_{0}$ is estimated by applying the formula for the mass diffusion coefficient of gases [29] and then using Eq. 6. In the case of the hydrogen molecule, for example, this procedure yields $z_{0} \approx 1$. It should be noted that, although the optical and the mass diffusion coefficients do not have the same physical significance, and therefore are not identical, they have the same order of magnitude. Indeed, $z_{0}=1$ is very close to the value extracted by fitting a Galatry function to the hydrogen spectra recorded at $8075 \mathrm{~cm}^{-1}$ [27]. Finally, we set the mirror reflectivity $R_{m}=0.9997$, temperature $T=298 \mathrm{~K}$ and the center frequency $v_{0}=3000 \mathrm{~cm}^{-1}$. Table 1 just summarizes all the above parameters that are set once and for all.

(i) Let us start considering a molecular transition with a very weak line strength $S=1 \cdot 10^{-27}$ $\mathrm{cm} /$ molec and set $z_{0}=1 \mathrm{~atm}^{-1}$. First, we simulate the corresponding line shape by means of Eq. 7 for different values of pressure. Then, from each spectrum we extract the half-width at half maximum (HWHM). The result of this procedure, shown in Fig. 1 (empty circles), gives back the typical behaviour: the line width approaches the Doppler one at low pressures, then a marked Dicke- 
narrowing manifests itself till a minimum value for the HWHM is reached; after this, the line width starts to increase again according to pressure broadening. The corresponding spectral profiles, as recorded by off-axis ICOS, are simulated by use of Eq. 9 for four different values of the cavity length $L$. The obtained HWHM values are plotted in Fig. 1 ( $L=25 \mathrm{~cm}$, rhombs), $(L=50 \mathrm{~cm}$, squares), ( $L=75 \mathrm{~cm}$, stars), ( $L=125 \mathrm{~cm}$, triangles). In this case, as already pointed out in a previous paper [31], a significant instrumental broadening of the lines is observed, which becomes more and more pronounced as the cavity length (and so the gain) increases. Another interesting feature is that, at the same time, the point of minimum width moves towards lower pressures, resulting in a shrinkage of the Dicke-narrowing pressure range. As an example, Fig. 1b shows the line shapes calculated for $p=1 \mathrm{~atm}$ both for the pure Galatry function (Eq. 8) and the cavity-recorded profiles (Eq. 9). A similar effect is found as the mirror reflectivity is changed.

(ii) Now we address the issue of a correct fitting procedure for accurate extraction of the line parameters which characterize the Galatry profiles, namely the $z_{0}$ parameter. For this purpose, we set $L=50 \mathrm{~cm}$ and $z_{0}=1 \mathrm{~atm}^{-1}$. Then, the off-axis ICOS molecular profiles, simulated by Eq. 9 for different pressures, are exported in the form of spectra recorded by discrete data points. For each pressure value, the corresponding spectrum is fitted directly with the Galatry function (Eq. 8) by a nonlinear least-squares procedure (Matlab Software). This gives the best-fit determination $z_{f i t}$ for $\mathrm{z}_{0}$, all the other parameters being fixed. The result of this procedure is summarized in Fig. 2a which shows, as a function of the gas pressure, the discrepancy $\left(z_{0}-z_{f i t}\right) / z_{0}$. This demonstrates that, neglecting the effects of cavity broadening can lead to errors of several tens percent in the determination of Galatry-lineshape parameters from the experimental data. The fitting curves together with the corresponding simulated spectra are also shown for the lowest and highest pressure (Fig. 2b). In order to investigate the extent of such fit discrepancy on the $z_{0}$ magnitude, the same procedure is repeated for a larger value $\left(z_{0}=2 \mathrm{~atm}^{-1}\right)$. Results are summarized in Figs. 3, showing an even more marked deviation. Of course, when Eq. 9 is used, the fitting procedure rapidly converges to the $z_{0}$ value set in the simulation. In this case, however, a sufficiently accurate knowledge of the cavity parameters $\left(R_{m}\right.$ and $\left.L\right)$ is needed. For this purpose, a previous determination of both the free spectral range $\left(F S R=\frac{c}{2 L}\right)$ and the ring-down time $\left(\tau=\frac{L}{c\left(1-R_{m}\right)}\right)$ for the empty cavity must be carried out. The relative precision and accuracy routinely achieved from these measurements are of the order of $10^{-5}$ and $10^{-7}$ for $L$ and $R_{m}$ respectively. When taken into account in the fitting procedure, such uncertainty levels are found to have practically no influence at all on the extracted parameter values $\left(z_{0}\right.$ in this case) over the whole pressure range. 
(iii) Finally, we find that, for higher molecular line strengths, the Dicke-narrowing effect may be completely hidden by the off-axis ICOS transfer function. This is shown, for $S=1 \cdot 10^{-26}$ $\mathrm{cm} / \mathrm{molec}$, in Fig. $4 \mathrm{a}\left(L=50 \mathrm{~cm}, z_{0}=1 \mathrm{~atm}^{-1}\right)$ where a flex around $p=1 \mathrm{~atm}$ seems to take the place of the minimum. Examples of spectral lineshapes for two different pressure values, $(p=0.1 \mathrm{~atm}$ and $p=1 \mathrm{~atm}$ respectively), are shown in Fig. $4 \mathrm{~b}$, where the pure Galatry function (dashed line) is compared to the simulated cavity-output profile (continuous line).

In conclusion, we have presented a detailed study of the characteristics of spectral lineshapes measured in Dicke narrowing regime by off-axis ICOS. For this purpose, we have explicitly included the Galatry profile describing the absorption coefficient into the cavity transfer function. From the simulated spectra we have extracted some interesting features for different experimental parameters. The first apparent effect is that the collisional narrowing pressure range shrinks as the cavity length increases due to the interplay between instrumental broadening and gain enhancement. Of course, similar results are found when the cavity gain is varied by changing the mirror reflectivity. For higher molecular absorption levels, realized for example when considering stronger transitions, the Dicke narrowing region is even predicted to disappear. We have also dealt with the issue of a correct fitting procedure of the recorded spectral profiles. To this aim, the simulated spectra have been fitted directly with a Galatry function, i.e. neglecting the cavity transfer function, as it is usually done in literature. In this way, we have quantified the resulting discrepancies between the best-fit parameters and those used in simulations. In future, investigation reported here could be extended to the case of a speed-dependent Voigt profile, which, as recently shown in [32, 33], describes more accurately the high pressure regime.

\section{References}

[1] P.A. Martin, Chem. Soc. Rev. 31, 201 (2002).

[2] U. Fink, M.J.S. Belton, J. Atmos. Sci. 26, 952 (1969).

[3] R.L. Armstrong, Appl. Opt. 14, 56 (1975).

[4] V.A. Krasnopolsky, P.D. Feldman, Science 294, 1914 (2001).

[5] B.E.J. Pagel, J. Phys. B: At. Mol. Phys. 4, 279 (1971).

[6] G. Sonnabend, D. Wirtz, R. Schieder, P.F. Bernath, Solar Physics 233, 205 (2006).

[7] R.H. Dicke, Phys. Rev. 89, 472 (1953).

[8] L. Galatry, Phys. Rev. A 135, 1218 (1964).

[9] M. Nelkin, A. Ghatak, Phys. Rev. A 135, 4 (1964). 
[10] F. Herbert, J. Quant. Spectrosc. Radiat. Transfer 14, 943 (1974).

[11] P.L. Varghese, R.K. Hanson, Appl. Opt. 23, 2376 (1984).

[12] J. Reid, A.R.W. McKellar, Phys. Rev A 18, 224 (1978)

[13] A.R.W. McKellar and T. Oka, Can. J. Phys. 56, 1315 (1978).

[14] S. Bhattacharya, A. Hazra, P.N. Ghosh, J. Mol. Structure 327, 139 (1994).

[15] S-I Chou, D.S. Baer, R.K. Hanson, J. Mol. Spectr. 199, 123 (1999).

[16] L. Moretti, A. Sasso, L. Gianfrani, and R. Ciurlo, J. Mol. Spectr. 205, 20 (2001).

[17] D. Lisak, G.. Rusciano, and A. Sasso, J. Mol. Spectr. 227, 162 (2004).

[18] P. De Natale, M. Bellini, M. Inguscio, G. Buffa, O. Tarrini, J. Mol. Spectr. 163, 510 (1994).

[19] D.A. Shapiro, A.D. May, Phys. Rev. A 63, 012701 (2000)

[20] R. Ciurlo, D. Lisak, J. Szudy, Phys. Rev. A 66, 032701 (2002)

[21] V.G. Avetisov, and P. Kauranen, Appl. Opt. 35, 4705 (1996).

[22] J.B. Paul, L. Lapson, J. Anderson, Appl. Opt. 40, 4904 (2001).

[23] D.S. Baer, J.B. Paul, M. Gupta, A. O’Keefe, Appl. Phys. B 75, 261 (2002).

[24] V.L. Kasyutich, C.E. Canosa-Mas, C. Pfrang, S. Vaughan, R.P. Wayne, Appl. Phys. B 75, 755 (2002).

[25] Y.A. Bakhirkin, A.A. Kosterev, C. Roller, R.F. Curl, F.K. Tittel, Appl. Opt. 43, 2257 (2004).

[26] P. Malara, P. Maddaloni, G. Gagliardi, P. De Natale, Opt. Express 14, 1304 (2006).

[27] M. Gupta, T. Owano, D.S. Baer, A. O’Keefe, Chem. Phys. Lett. 418, 11 (2006).

[28] J.O. Hirschfelder in "Molecular theory of gases and liquids", Wiley New York (1954).

[29] O. Moeschlin, E. Grycko: Experimental Stochastics in Physics. Springer-Verlag, Berlin, Heidelberg, New York 2006.

[30] G. Herzberg, Can. J. Phys. A28, 144 (1950).

[31] P. Maddaloni, G. Gagliardi, P. Malara, P. De Natale, J. Opt. Soc. Am. B 23, 1938 (2006).

[32] F. Rohart, L. Nguyen, J. Buldyreva, J.-M. Colmont, G. Wlodarczak, J. Mol. Spectr. 246, 213 (2007)

[33] F. Rohart, G. Wlodarczak, J.-M. Colmont, G. Cazzoli, L. Dore, C. Puzzarini, J. Mol. Spectr. 251, $282(2008)$

\section{Table and figure captions}

Table 1 - List of parameters which remain the same in all the numerical calculations. 
Fig. 1a-Calculated HWHM values vs. gas pressure. Eq. 8 is used to simulate the Galatry function (empty circles), while Eq. 9 yields the corresponding spectral profiles recorded by off-axis ICOS for four different cavity lengths (rhombs $\rightarrow 25 \mathrm{~cm}$, squares $\rightarrow 50 \mathrm{~cm}$, stars $\rightarrow 75 \mathrm{~cm}$, triangles $\rightarrow 125 \mathrm{~cm}$ ). Relative absorption in the considered pressure interval (0.1-3.5 atm) ranges from a few to several tens percent $(1-33 \%$ for $L=25 \mathrm{~cm}, 2-49 \%$ for $L=50 \mathrm{~cm}, 3-59 \%$ for $L=75 \mathrm{~cm}, 6-71 \%$ for $L=125$ $\mathrm{cm})$.

Fig $1 \mathrm{~b}$ - Example of spectral profiles, (normalized to peak value), calculated for $p=1 \mathrm{~atm}$ by use of Eq. 8 (dashed line) and Eq. 9 (continuous lines). The latter curves, ordered for increasing line widths, correspond to the four different cavity lengths considered in the text $(L=25,50,75,125 \mathrm{~cm})$.

Fig $2 \mathrm{a}$ - Percentage discrepancy $v s$. pressure between the parameter $z_{0}=1 \mathrm{~atm}^{-1}$ used in the simulation of the cavity-recorded spectral profiles and the value $\mathrm{z}_{f i t}$ extracted by fitting Eq. 8 to such line shapes.

Fig $2 \mathrm{~b}$ - Example of fitting procedure for $L=50 \mathrm{~cm}$ and $z_{0}=1 \mathrm{~atm}^{-1}$. First, Eq. 9 is used to simulate the cavity-recorded spectrum (normalized to peak value); then, by means of Eq. 8 the best-fit curve is found (continuous line) to extract $z_{\text {fit }}$, all the other parameters being fixed. Left (right) frame, calculated for $p=0.5 \mathrm{~atm}(p=2.75 \mathrm{~atm})$, corresponds to the first (last) point in Fig. $2 \mathrm{a}$. In the latter case a larger fit discrepancy, particularly visible in the wings, is found.

Fig $3 \mathrm{a}$ - Percentage discrepancy $v s$. pressure between the parameter $z_{0}=2 \mathrm{~atm}^{-1}$ used in the simulation of the cavity-recorded spectral profiles and the value $\mathrm{z}_{f i t}$ extracted by fitting Eq. 8 to such line shapes.

Fig $3 \mathrm{~b}$ - Example of fitting procedure for $L=50 \mathrm{~cm}$ and $z_{0}=2 \mathrm{~atm}^{-1}$. Left (right) frame, calculated for $p=0.5 \mathrm{~atm}(p=2.75 \mathrm{~atm})$, corresponds to the first (last) point in Fig. 3a. In the latter case a discrepancy as high as $70 \%$ is found in the determination of the $z_{0}$ parameter.

Fig 4a - Calculated HWHM values vs. gas pressure for a stronger molecular transition $\left(S=1 \cdot 10^{-26}\right.$ $\mathrm{cm} / \mathrm{molec}$ ). Like in Fig. 1, Eq. 8 is used to simulate the Galatry function (empty circles), while Eq. 9 yields the corresponding spectral profiles recorded by off-axis ICOS (filled circles). Now, relative absorption ranges from $19 \%(0.1 \mathrm{~atm})$ to $91 \%$ (3.5 atm). In this configuration, Dicke narrowing effect is completely hidden by the cavity transfer function. 
Fig. $4 \mathrm{~b}$ - Comparison between Galatry function (Eq. 8) and cavity-output line shape (Eq. 9) for $L=50 \mathrm{~cm}, z_{0}=1 \mathrm{~atm}^{-1}, S=1 \cdot 10^{-26} \mathrm{~cm} / \mathrm{molec}$. Left (right) frame corresponds to $p=0.1 \mathrm{~atm}$ ( $p=1 \mathrm{~atm}$ ). 


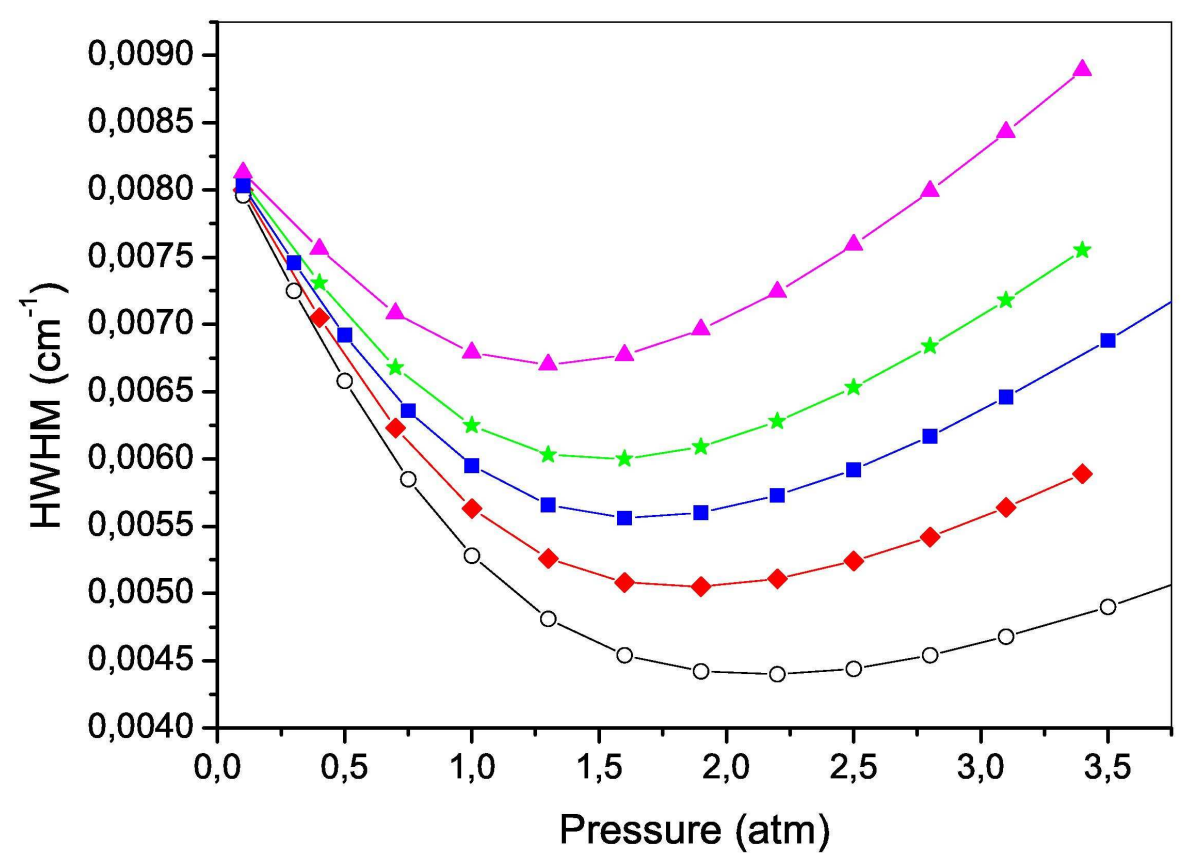

$106 \times 80 \mathrm{~mm}(600 \times 600$ DPI $)$

URL: http://mc.manuscriptcentral.com/tandf/tmph 
1

2

3

4

5

6

7

8

10

11

12

13

14

15

16

17

18

19

20

21

22

23

24

25

26

27

28

29

30

31

32

33

34

35

36

37

38

39

40

41

42

43

44

45

46

47

48

49

50

51

52

53

54

55

56

57

58

59

60

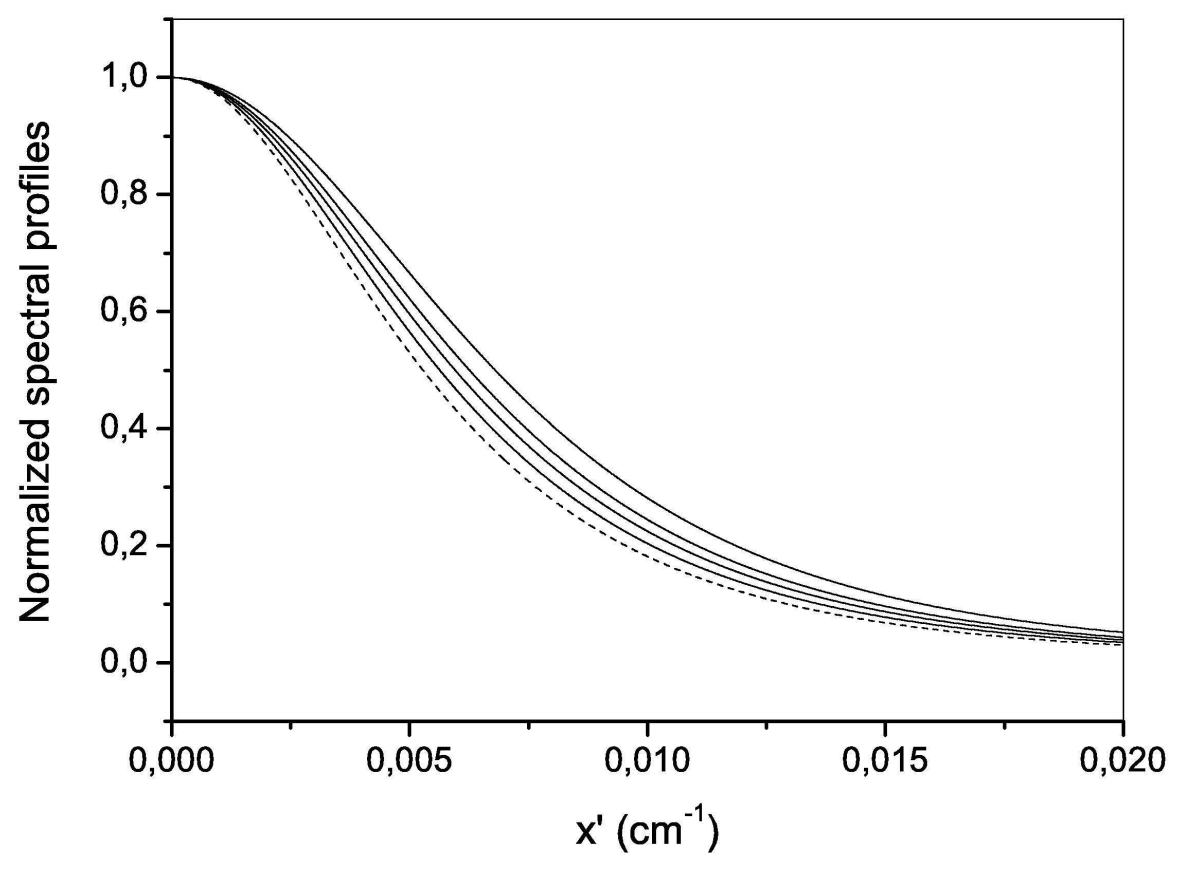

$107 \times 80 \mathrm{~mm}(600 \times 600$ DPI $)$ 


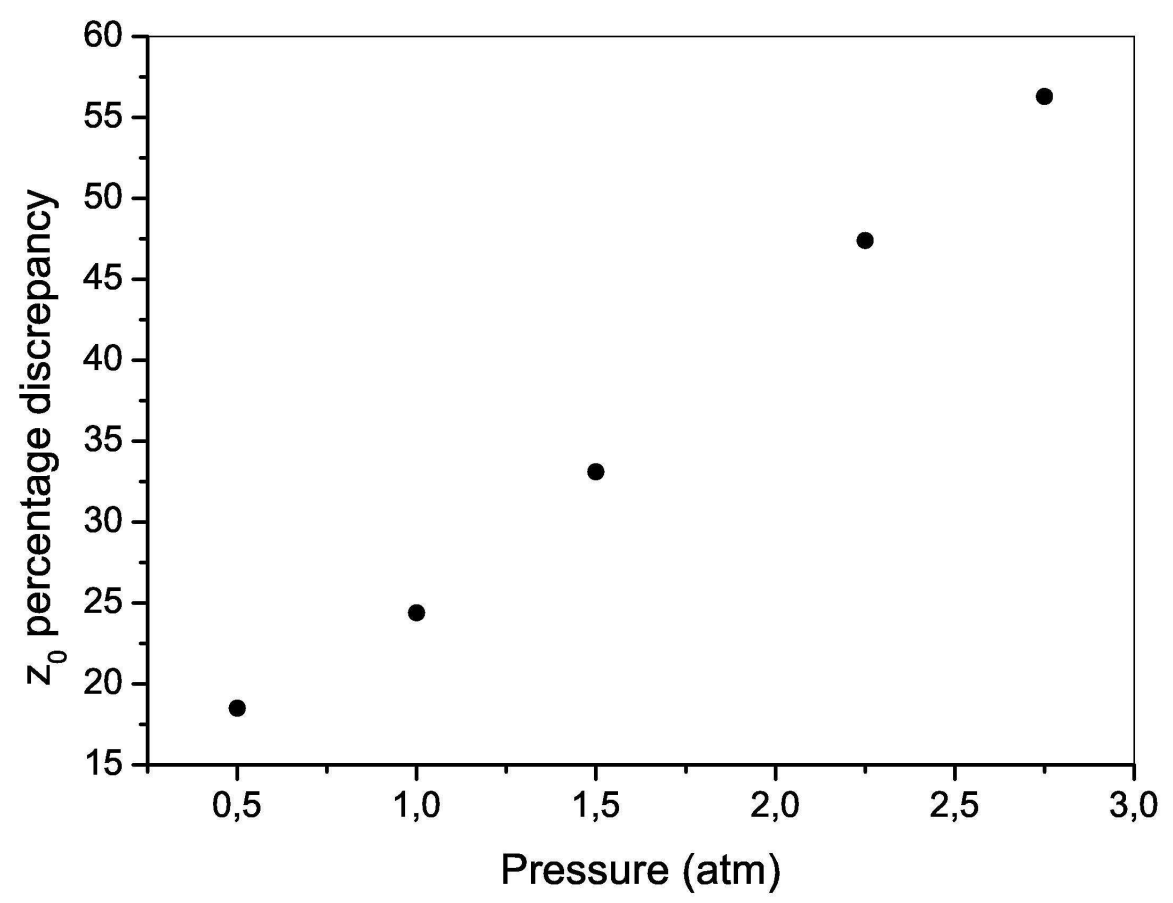

$103 \times 82 \mathrm{~mm}(600 \times 600 \mathrm{DPI})$

URL: http://mc.manuscriptcentral.com/tandf/tmph 


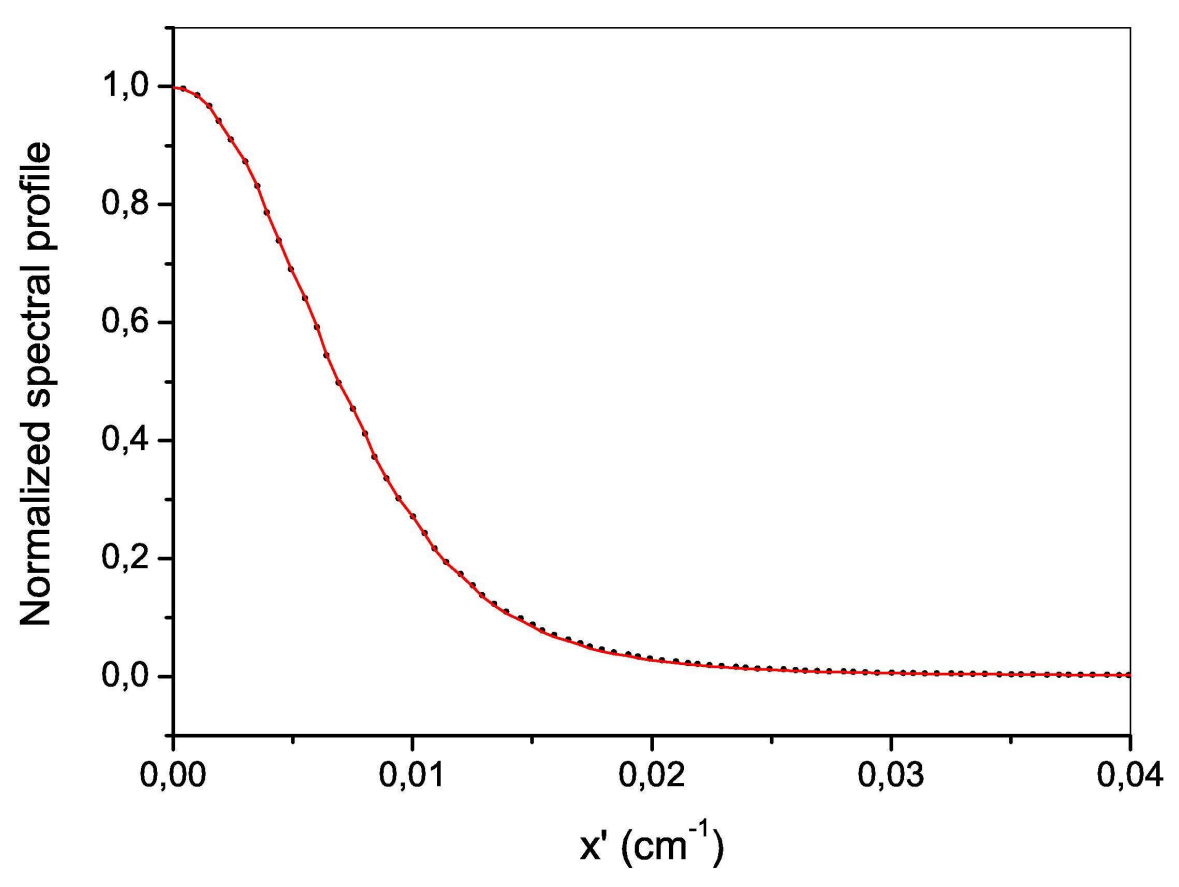

$106 \times 80 \mathrm{~mm}(600 \times 600 \mathrm{DPI})$

URL: http://mc.manuscriptcentral.com/tandf/tmph 


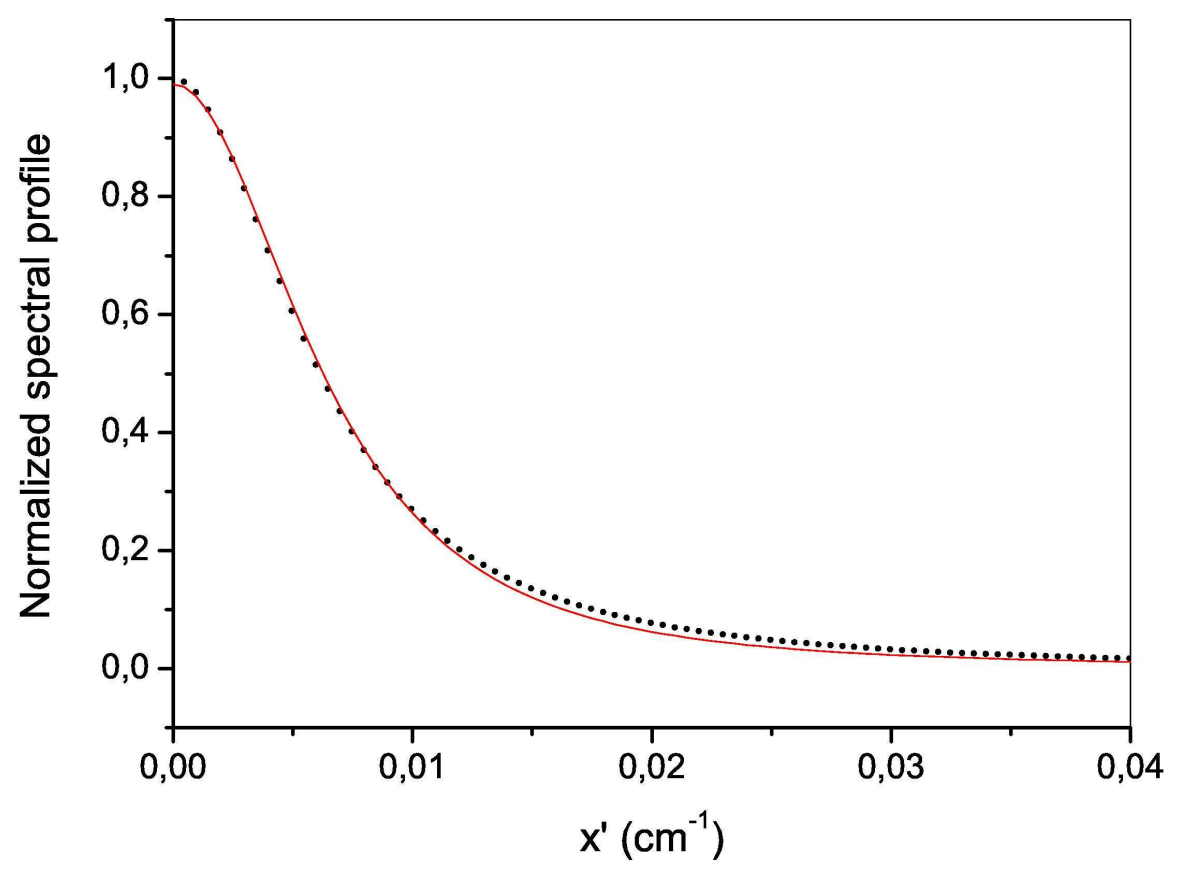

$106 \times 80 \mathrm{~mm}(600 \times 600 \mathrm{DPI})$ 


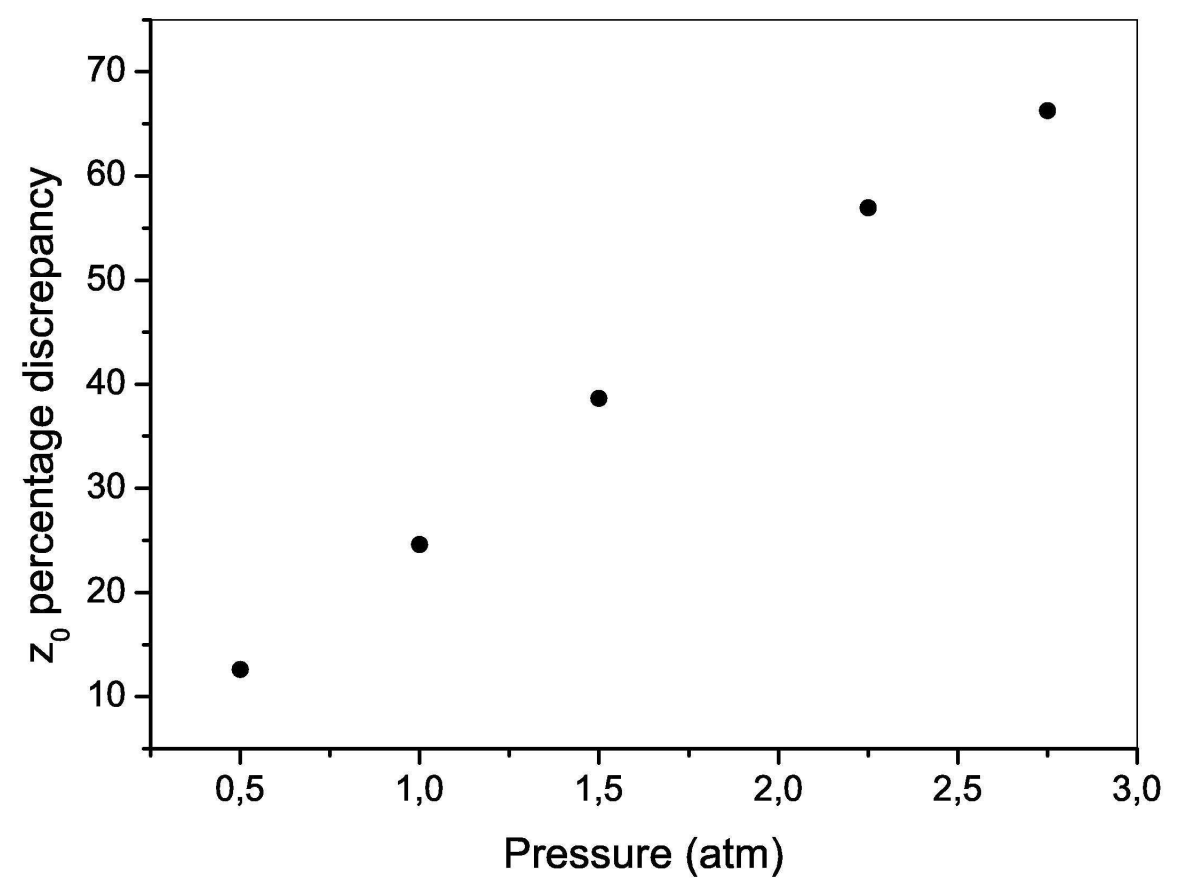

$103 \times 80 \mathrm{~mm}(600 \times 600 \mathrm{DPI})$ 


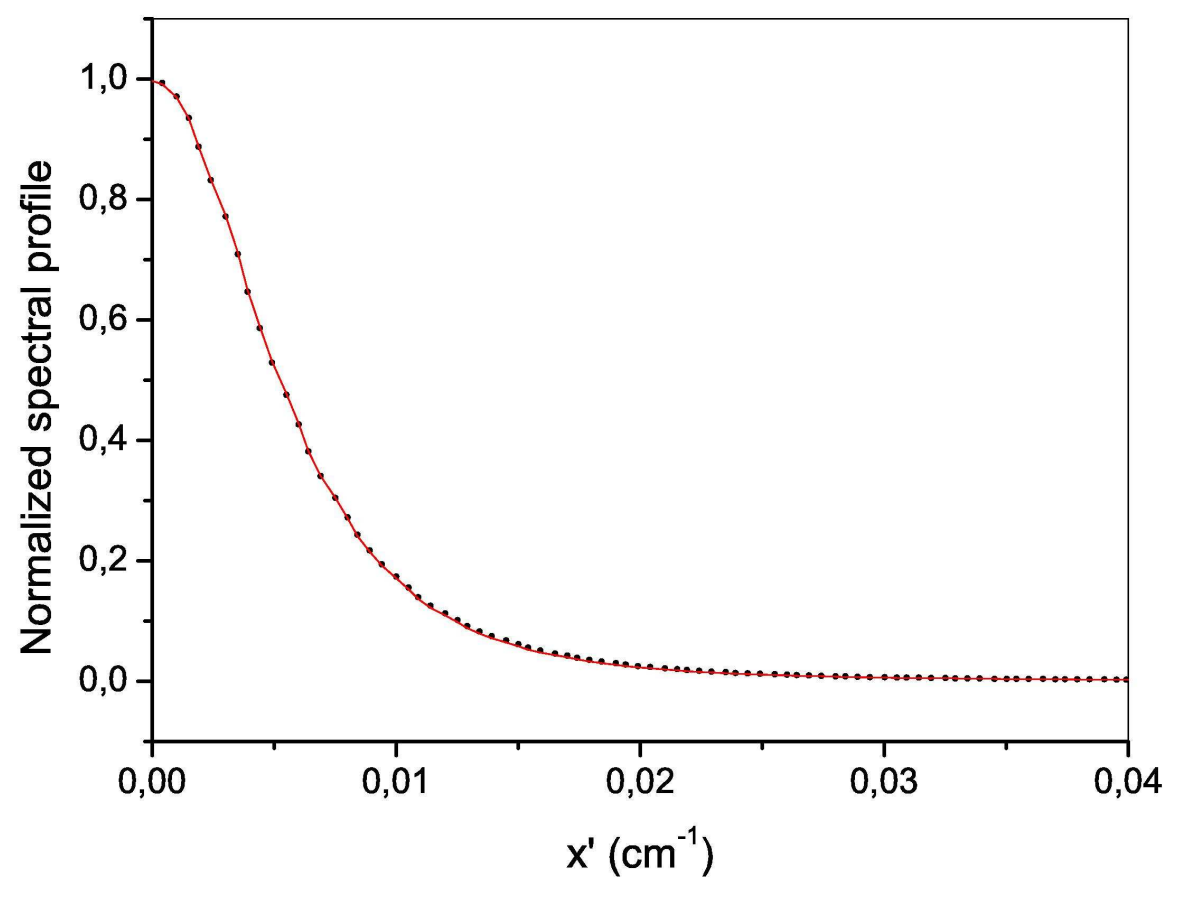

$104 \times 80 \mathrm{~mm}(600 \times 600 \mathrm{DPI})$

URL: http://mc.manuscriptcentral.com/tandf/tmph 
1

2

3

4

5

6

7

8

10

11

12

13

14

15

16

17

18

19

20

21

22

23

24

25

26

27

28

29

30

31

32

33

34

35

36

37

38

39

40

41

42

43

44

45

46

47

48

49

50

51

52

53

54

55

56

57

58

59

60

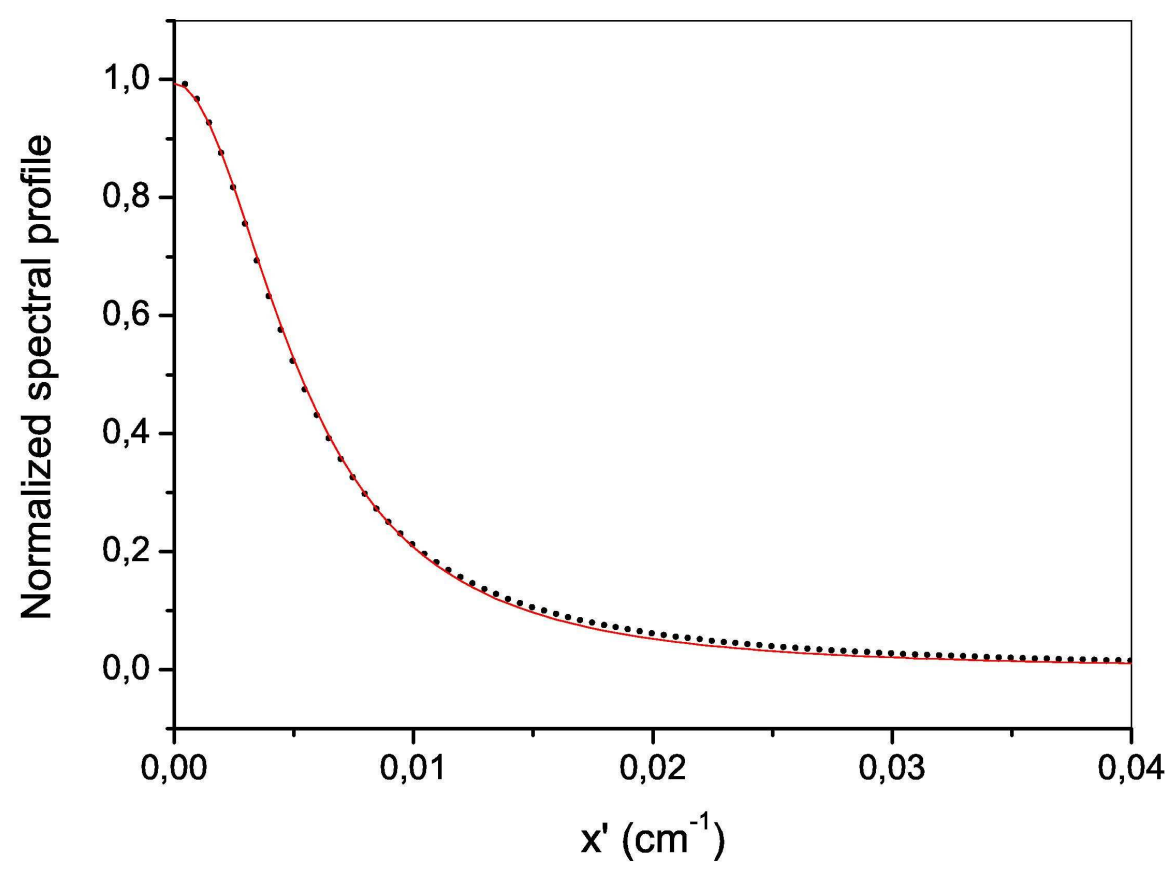

$106 \times 80 \mathrm{~mm}(600 \times 600 \mathrm{DPI})$ 


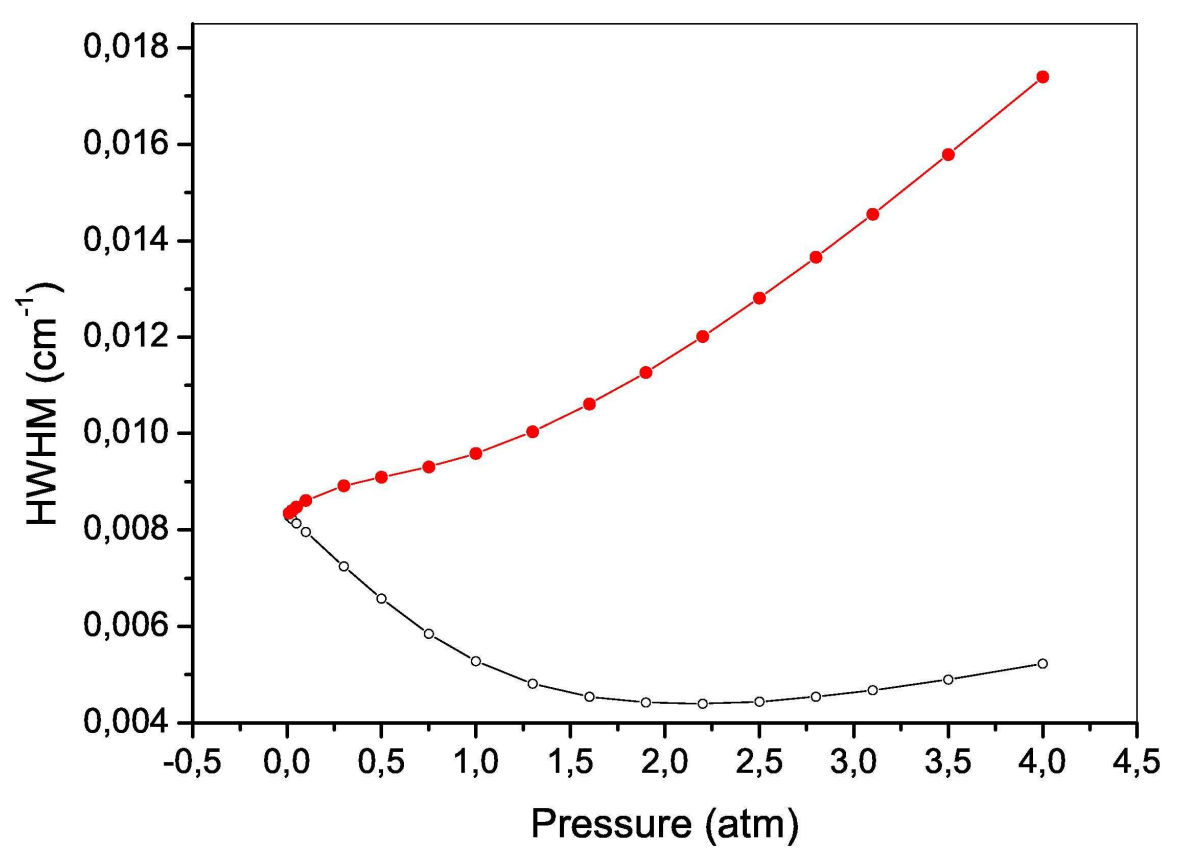

$107 \times 80 \mathrm{~mm}(600 \times 600 \mathrm{DPI})$ 
1

2

3

4

5

6

7

8

10

11

12

13

14

15

16

17

18

19

20

21

22

23

24

25

26

27

28

29

30

31

32

33

34

35

36

37

38

39

40

41

42

43

44

45

46

47

48

49

50

51

52

53

54

55

56

57

58

59

60

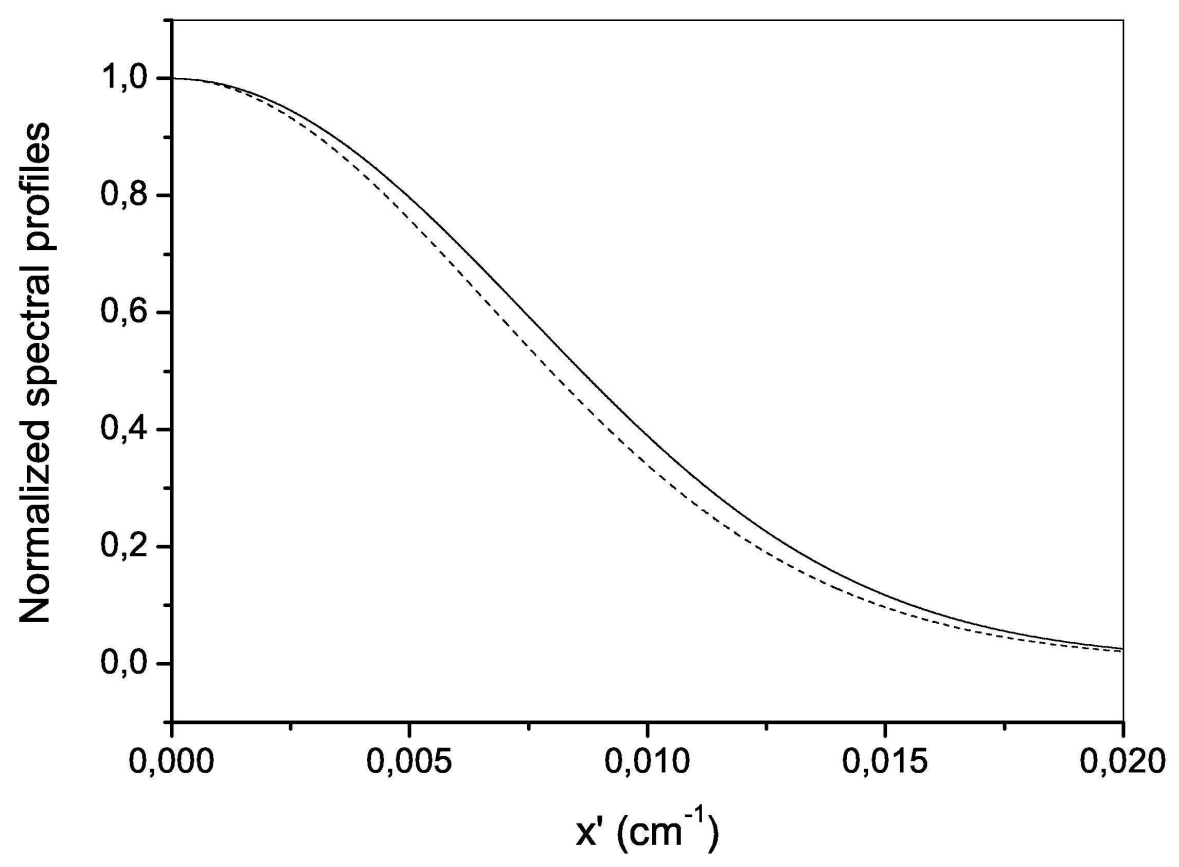

$107 \times 80 \mathrm{~mm}(600 \times 600 \mathrm{DPI})$ 


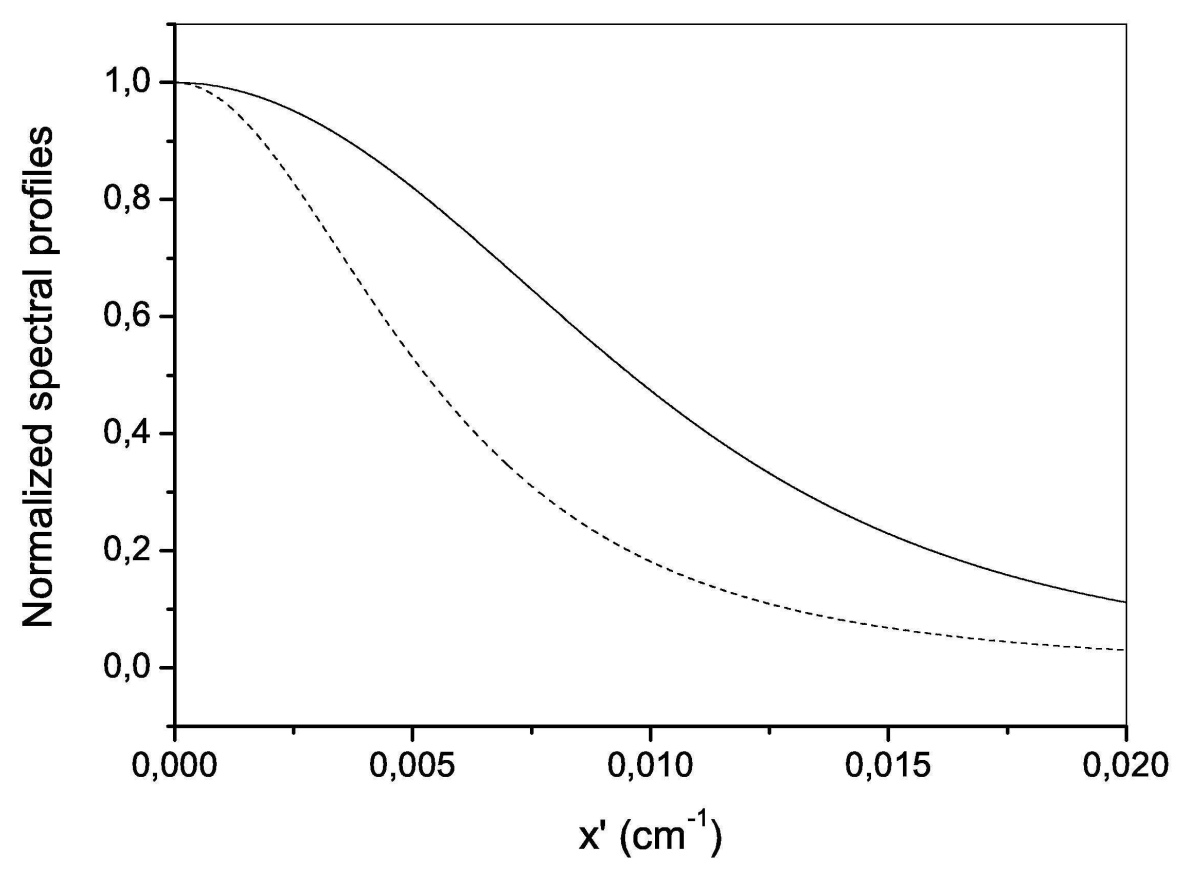

$107 \times 80 \mathrm{~mm}(600 \times 600$ DPI $)$

URL: http://mc.manuscriptcentral.com/tandf/tmph 


\begin{tabular}{|l|l|}
\hline$y_{0}$ & $0.1 \mathrm{~atm}^{-1}$ \\
\hline$\delta$ & $0.001 \mathrm{~cm}^{-1} \mathrm{~atm}^{-1}$ \\
\hline$\Delta v_{D}$ & $0.01 \mathrm{~cm}^{-1}$ \\
\hline$R_{m}$ & 0.9997 \\
\hline$T$ & $298 \mathrm{~K}$ \\
\hline$v_{0}$ & $3000 \mathrm{~cm}^{-1}$ \\
\hline
\end{tabular}

Table 1 - List of parameters which remain the same in all the numerical calculations. 


\section{Dear Editor,}

we thank the referee for appreciating our manuscript and for giving useful suggestions to improve it. In the following, we report specific answers to the questions raised by the reviewers. The corresponding changes in the revised manuscript are evidenced by using red text.

\section{$\underline{\text { Referee } 1}$}

1) The results of our investigation basically show that, in order to accurately retrieve the line parameters from the fit of the observed spectra, the cavity transfer function cannot be ignored. Of course, when Eq. 9 is used (rather than just the absorption profile given by Eq. 8), the fitting procedure rapidly converges to the $z_{0}$ value set in the simulation. When this method of analysis is adopted, the only request is a sufficiently accurate knowledge of the cavity parameters $\left(R_{m}\right.$ and $\left.L\right)$. For this purpose, a previous determination of both the free spectral range $\left(F S R=\frac{c}{2 L}\right)$ and the ring-down time $\left(\tau=\frac{L}{c\left(1-R_{m}\right)}\right)$ for the empty cavity must be carried out. Since relative precision of the order of $10^{-3}$ and $10^{-5}$ is routinely achieved for $\tau$ and FSR respectively, the resulting relative uncertainty is of the order of $10^{-5}$ for $L$ and $10^{-7}$ for $R_{m}$. Then, we have checked, over the whole pressure range, that such uncertainty levels practically do not affect the value of the parameters extracted from the fitting procedure ( $z_{0}$ in our case). We clarify this point by adding the following sentence (Pag. 5, Line 8 from the bottom):

Of course, when Eq. 9 is used, the fitting procedure rapidly converges to the $z_{0}$ value set in the simulation. In this case, however, a sufficiently accurate knowledge of the cavity parameters $\left(R_{m}\right.$ and $\left.L\right)$ is needed. For this purpose, a previous determination of both the free spectral range $\left(F S R=\frac{c}{2 L}\right)$ and the ring-down time $\left(\tau=\frac{L}{c\left(1-R_{m}\right)}\right)$ for the empty cavity must be carried out. The relative precision and accuracy routinely achieved from these measurements are of the order of $10^{-5}$ and $10^{-7}$ for $L$ and $R_{m}$ respectively. When taken into account in the fitting procedure, such uncertainty levels are found to have practically no influence at all on the extracted parameter values ( $z_{0}$ in this case) over the whole pressure range.

2) A thorough analysis of the molecular lineshape function which better describes the physics of collisions for each pressure regime is beyond the scope of the current investigation. The latter is, in fact, mainly intended to point out the influence of the transfer function of the widely used off-axis integrated cavity output spectroscopy technique on the interpretation of the recorded spectra. Indeed, we have shown that such effects are significant at low and intermediate gas pressures too, where the Galatry function describes the molecular line profile pretty well. Of course, as suggested by the referee, our study could be refined by including in the simulation the most appropriate lineshape function for each collisional regime (pressure range, colliding species...). We clarify this by adding the following sentence in the paper conclusions (last three lines) and two new references:

In future, investigation reported here could be extended to the case of a speed-dependent Voigt profile, which, as recently shown in $[32,33]$, describes more accurately the high pressure regime. 
[32] F. Rohart, L. Nguyen, J. Buldyreva, J.-M. Colmont, G. Wlodarczak, J. Mol. Spectr. 246, 213 (2007)

[33] F. Rohart, G. Wlodarczak, J.-M. Colmont, G. Cazzoli, L. Dore, C. Puzzarini, J. Mol. Spectr. 251, 282 (2008)

\section{Referee 2}

1) We now express the line strength in $\mathrm{cm} /$ molec across the whole paper. 